\title{
N-Type Carbon-Nanotube MOSFET Device Profile Optimization for Very Large Scale Integration
}

\author{
Yanan Sun ${ }^{\dagger}$ and Volkan Kursun \\ Department of Electronic and Computer Engineering, The Hong Kong University of Science and Technology, Clear Water Bay, \\ Kowloon, Hong Kong
}

\begin{abstract}
Carbon-nanotube metal oxide semiconductor field effect transistor (CN-MOSFET) is a promising future device candidate. The electrical characteristics of $16 \mathrm{~nm} \mathrm{~N}$-type CN-MOSFETs are explored in this paper. The optimum $\mathrm{N}$-type CN-MOSFET device profiles with different number of tubes are identified for achieving the highest on-state to off-state current ratio $\left(\mathrm{I}_{\text {on }} / \mathrm{I}_{\text {off }}\right)$. The influence of substrate voltage on device performance is also investigated in this paper. Tradeoffs between subthreshold leakage current and overall switch quality are evaluated with different substrate bias voltages. Technology development guidelines for achieving high-speed, low-leakage, area efficient, and manufacturable carbon nanotube integrated circuits are provided.
\end{abstract}

Keywords: Carbon nanotube array, Lower subthreshold leakage, Higher performance, Substrate bias, Charge screening effect

\section{INTRODUCTION}

As the complementary metal-oxide semiconductor (CMOS) technology progresses into the $22 \mathrm{~nm}$ regime, the fundamental limitations of silicon are felt stronger. New superior materials are needed to achieve higher performance and scalable CMOS integrated circuits in the future. Carbon-nanotube $(\mathrm{CN})$ transistor is a promising candidate for replacing silicon metal oxide semiconductor field effect transistor (Si-MOSFET) [1-6]. CN transistors display desirable characteristics such as elastic carrier scattering, ballistic carrier transport, and smaller device footprint (area) as compared to conventional Si-MOSFETs [1].

Two types of CN transistors have recently attracted significant attention in the literature: Schottky Barrier (SB) controlled switch (CN-SBFET) and MOSFET-like switch (CN-MOSFET) [1-6]. CNSBFETs are formed by directly attaching the intrinsic nanotube channels to the metal source/drain contacts. CN-SBFETs show ambipolar carrier transport. Alternatively, the CN-MOSFET displays unipolar behavior with heavily doped source and drain extension regions. A coaxial gated CN-SBFET and a CN-MOSFET are compared in [2]. CN-MOSFETs produce higher on-state and lower leakage currents. By providing a significantly higher $\mathrm{I}_{\text {on }} / \mathrm{I}_{\text {off }}$ ratio, CN-MOSFETs display better scalability as compared to $\mathrm{CN}$ -

\footnotetext{
${ }^{\dagger}$ Author to whom all correspondence should be addressed: E-mail: eesyun@ust.hk
}

SBFETs. CN-MOSFET is the focus of the study presented in this paper due to the superior performance as compared to the $\mathrm{CN}$ SBFET.

The structure of a planar-gated N-type CN-MOSFET is shown in Fig. 1. The CNs are heavily doped with donors $\left(\mathrm{n}^{+}\right)$in the source and drain extension regions in an N-type CN-MOSFET. The nanotubes are undoped under the gate. The cross-sectional view of a CN-MOSFET is shown in Fig. 2. Multiple nanotubes are placed in parallel to form an array that produces sufficient current with a CN-MOSFET.

Inspired by the developments in $\mathrm{CN}$ related research, publications on novel circuit techniques for very large scale integration (VLSI) of CN-MOSFETs have recently started to appear in literature [7-11]. All of these studies are based on Stanford CN-MOSFET HSPICE compact model [12]. A multithreshold voltage six-transistor (6-T) static random access memory (SRAM) cell using CN-MOSFETs is presented by Lin et al. in [7] to provide higher performance as compared to the $32 \mathrm{~nm}$-Si-MOSFET based memory cells. The characteristics of 6-T CN-MOSFET and Si-MOSFET SRAM cells are compared by Moradinasab et al. in [8] assuming a $32 \mathrm{~nm}$ CMOS technology. A new CN-MOSFET SRAM cell is proposed by Ebrahimi and Afzali-Kusha in [9] to enhance the read static noise margin as compared to a conventional Si-MOSFET memory cell. A novel eight-transistor CN-MOSFET SRAM cell is proposed by Kim et al. in [10] to lower the dynamic switching power consumption 


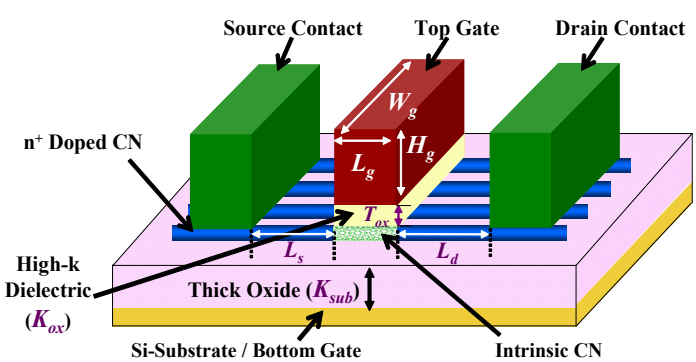

Fig. 1. Three-dimensional view of a planar-gated N-type CN-MOSFET.

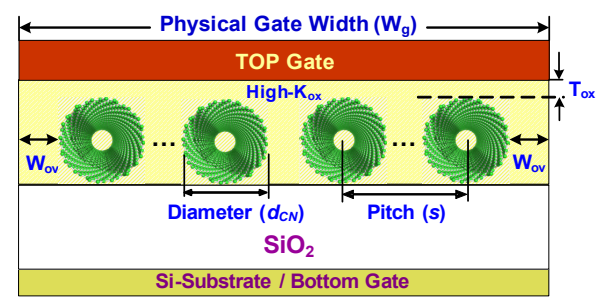

Fig. 2. Cross-sectional view of a multi-tube CN-MOSFET. $W_{o v}$ is the overhang of the gate from the edge of $\mathrm{CN}$ array.

as compared to silicon FinFET memory circuits. A new ternary inverter is proposed by Lin et al. in [11] for lower power consumption and higher performance with the CN-MOSFET technology. The conclusions of these recent publications are however difficult to reproduce and verify. Unlike a Si-MOSFET, device size is not determined merely by the physical gate width and channel length in a CN-MOSFET. The complex relationship between device sizing and performance due to the unique material properties and nanotube array structure of a CN-MOSFET is typically overlooked in these previous publications. Device and circuit design options such as transistor sizing (number of tubes), carbon nanotube array physical structure (nanotube diameters and pitch), and choice of substrate voltage are not discussed in sufficient detail.

A thorough understanding and a careful optimization of the device structure are essential to be able to develop high-performance integrated circuits with $\mathrm{CN}$ transistors. A p-channel CNMOSFET device profile optimization study for achieving highspeed carbon nanotube integrated circuits is presented in [13]. Alternatively, the electrical characteristics of $16 \mathrm{~nm} \mathrm{~N}$-type CNMOSFETs are explored from a circuit designer's point of view in this paper. The purpose of this paper is to bridge the fledgling CN-MOSFET based novel circuit development and VLSI efforts to the underlying emerging $\mathrm{CN}$ technology. The optimization study is based on the Stanford CN-MOSFET HSPICE compact model [12]. The dependence of device performance on the physical geometry of carbon nanotubes is presented. The optimum high-performance device profiles that provide the maximum $\mathrm{I}_{\mathrm{on}} /$ $\mathrm{I}_{\text {off }}$ ratio are identified. Technology development and utilization guidelines are provided to achieve high-speed, low-leakage, area efficient, and manufacturable integrated circuits with CN field effect transistors.

The paper is organized as follows. The performance-critical parameters in the physical structure of a CN-MOSFET are highlighted in Section 2. The optimization results with technology development and utilization guidelines for high-performance $16 \mathrm{~nm}$ N-type CN-MOSFETs are presented in Section 3. Finally, some conclusions are offered in Section 4.

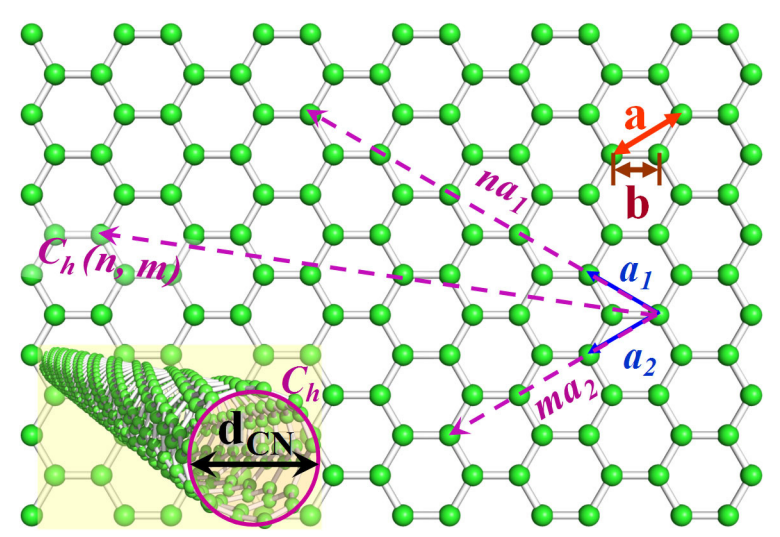

Fig. 3. The structure of a carbon nanotube with chirality vector $C_{h}$ (n, $\mathrm{m})$. The carbon nanotube is formed by rolling up a sheet of graphene along the chirality vector $C_{h}$. The carbon to carbon inter-atomic distance along the hexagonal lattice is $\mathrm{b} \approx 1.44 \AA$. The lattice unit vector is characterized by $\left(a_{1}, a_{2}\right)$. The inter-atomic distances along the lattice unit vectors are $\mathrm{a}=\left|a_{1}\right|=\left|a_{2}\right|=\sqrt{3} b=2.49 \AA . C_{h}=\mathrm{n} \cdot a_{1}+\mathrm{m} \cdot a_{2}$.

\section{IMPORTANT CN-MOSFET PARAMETERS}

Important device parameters for modeling and performance characterization of CN-MOSFETs are highlighted in this section. The relationships among $\mathrm{CN}$ diameter, energy bandgap, $\mathrm{I}_{\mathrm{on}}$, and $\mathrm{I}_{\text {off }}$ in a CN-MOSFET are explained in Section 2.1. The distinctions among the physical gate width, physical channel width, and effective channel width in a CN-MOSFET are described in Section 2.2. The important set of physical parameters from the $16 \mathrm{~nm}$ CN-MOSFET technology considered in this performance optimization study is presented in Section 2.3.

\subsection{Carbon-nanotube diameter}

The diameter of a single-walled CN (SWCN) is specified by the chirality vector $C_{h}(\mathrm{n}, \mathrm{m})$ as shown in Fig. 3 . Both $\mathrm{n}$ and $\mathrm{m}$ are positive integers. SWCN is metallic if $|\mathrm{n}-\mathrm{m}|$ is an integer multiple of 3 [14]. Alternatively, if $|\mathrm{n}-\mathrm{m}|$ is not an integer multiple of 3 , SWCN is a semiconductor [14]. The diameter $\left(d_{C N}\right)$ of a singlewalled CN (SWCN) with chirality vector $(n, m)$ is [14]

$$
d_{C N}=\frac{a * \sqrt{n^{2}+n m+m^{2}}}{\pi}
$$

where $a(2.49 \AA)$ is the carbon to carbon inter-atomic distance along the lattice unit vectors $a_{1}$ and $a_{2}$ as shown in Fig. 3.

The energy bandgap $\left(E_{g}\right)$ of carbon nanotube is inversely proportional to the nanotube diameter [14]. The diameter therefore influences both on-state and off-state currents. Appropriate choice of CN diameter is critical to achieve a high speed and energy efficient electrical switch.

\subsection{Width of a CN-MOSFET}

The channel is a doped single piece of silicon extending between the source and drain terminals in a Si-MOSFET. The gate width is essentially the length of the (polysilicon or metal) gate that overlaps the channel area perpendicular to the direction of current flow between the source and drain in a Si-MOSFET. The definition and measurement of the gate width are however different in a CN-MOSFET. Unlike a Si-MOSFET, the channel of a CN-MOSFET is composed of an array of discrete nanotubes 
Table 1. Set of process parameters for $16 \mathrm{~nm}$ N-type CN-MOSFET.

\begin{tabular}{|c|c|c|}
\hline Process parameters & Default values for $32 \mathrm{~nm}$ CN-MOSFET [12] & Fixed values for $16 \mathrm{~nm}$ CN-MOSFET \\
\hline Supply voltage & $0.9 \mathrm{~V}$ & $0.7 \mathrm{~V}$ \\
\hline Physical channel length $\left(\mathrm{L}_{\mathrm{g}}\right)$ & $32 \mathrm{~nm}$ & $16 \mathrm{~nm}$ \\
\hline Length of doped CN source-side $\left(\mathrm{L}_{\mathrm{s}}\right)$ & $32 \mathrm{~nm}$ & $16 \mathrm{~nm}$ \\
\hline Length of doped CN drain-side $\left(\mathrm{L}_{\mathrm{d}}\right)$ & $32 \mathrm{~nm}$ & $16 \mathrm{~nm}$ \\
\hline Dielectric constant of bottom gate $\left(\mathrm{K}_{\mathrm{sub}}\right)$ & $\mathrm{SiO}_{2}(4)$ & $\mathrm{SiO}_{2}(4)$ \\
\hline Thick oxide & $10 \mu \mathrm{m}$ & $10 \mu \mathrm{m}$ \\
\hline Top gate dielectric material $\left(\right.$ High- $\left.\mathrm{K}_{\mathrm{ox}}\right)$ & $\mathrm{HfO}_{2}(16)$ & $\mathrm{ZrO}_{2}(25)$ \\
\hline Thickness of top gate dielectric $\left(\mathrm{T}_{\mathrm{ox}}\right)$ & $4 \mathrm{~nm}$ & $3 \mathrm{~nm}$ \\
\hline Height of gate/source/drain contact & $64 \mathrm{~nm}$ & $60 \mathrm{~nm}$ \\
\hline Flatband voltage of N-type CN-MOSFET $\left(\mathrm{V}_{\mathrm{fbn}}\right)$ & 0 & 0 \\
\hline Fermi level of $\mathrm{n}^{+}$doped source/drain $\mathrm{CN}$ region $\left(\mathrm{E}_{\mathrm{fo}}\right)$ & $0.6 \mathrm{eV}(\sim 0.8 \%)$ (uniformly distributed) & $0.6 \mathrm{eV}(\sim 0.8 \%)$ (uniformly distributed) \\
\hline Mean free path: intrinsic CN & $200 \mathrm{~nm}$ & $200 \mathrm{~nm}$ \\
\hline Mean free path: doped CN & $15 \mathrm{~nm}$ & $15 \mathrm{~nm}$ \\
\hline Interconnect capacitance & $0.213 \mathrm{fF} / \mu \mathrm{m}$ & $0.3 \mathrm{fF} / \mu \mathrm{m}^{\mathrm{a}}$ \\
\hline
\end{tabular}

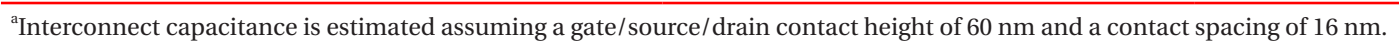

separated by insulator as illustrated in Fig. 2. The gate width of a CN-MOSFET can be therefore defined in three different ways.

The total area of a CN-MOSFET is determined by the physical gate width $\left(W_{g}\right)$ as shown in Fig. 2 . $W_{g}$ is determined by the intertube pitch, the number of tubes, the diameter of CNs, and the gate overhangs beyond the carbon nanotubes at the two ends of the channel. $W_{g}$ is

$$
W_{g}=s *(N-1)+d_{C N}+2 * W_{o v}
$$

where $\mathrm{N}$ is the number of tubes in a CN-MOSFET. Inter-tube pitch $(s)$ is the distance between the centers of two adjacent nanotubes that form the channel of a CN-MOSFET. $W_{o v}$ is the overhang width of the gate from the edge of the $\mathrm{CN}$ array as shown in Fig. 2. $W_{o v}$ helps to lower the probability of leaving uncovered carbon nanotubes in the channel area due to the misalignments of the gate during fabrication. In a Si-MOSFET, the overhang width of the gate from the edge of the active region is typically $2 \lambda$ [15]. Assuming a similar photolithographic manufacturing process for $\mathrm{CN}$ transistors, $W_{o v}$ at each end is assumed to be $2 \lambda(16 \mathrm{~nm})$ in this study as shown in Fig. 2 .

A single $\mathrm{CN}$ typically provides a limited amount of current. An array of multiple nanotubes is therefore necessary to be able to produce sufficient drain-to-source current in a CN-MOSFET. The physical channel width $\left(W_{c h}\right)$ is the total accumulated diameters of the nanotubes that form the channel in a CN-MOSFET. $W_{c h}$ is

$$
W_{c h}=N * d_{C N}
$$

The electric field lines emerging from the gate terminal terminate on the CNs that form the channel. These electric field lines penetrating into the channel area are screened by the neighboring insulated nanotubes. When a positive voltage is applied on the gate terminal of an N-type CN-MOSFET, the charge induced on the CNs interact as illustrated in Fig. 4. The gate-to-channel capacitance and the resulting current produced by a nanotube are reduced due to enhanced screening effect with decreased inter-tube spacing (pitch). Charge screening reduces the effective width of the channel, thereby degrading the device current $[16,17]$. Considering the charge screening effect, the effective channel width $\left(W_{\text {eff }}\right)$ of a CN-MOSFET is

$$
W_{e f f}=N * d_{C N} * \alpha
$$

where $\alpha$ is the screening effect coefficient $(0<\alpha \leq 1)$. When there is only one tube per gate, $\alpha=1$. For $N>1, \alpha$ is primarily (a)

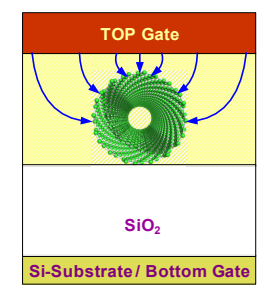

(b)

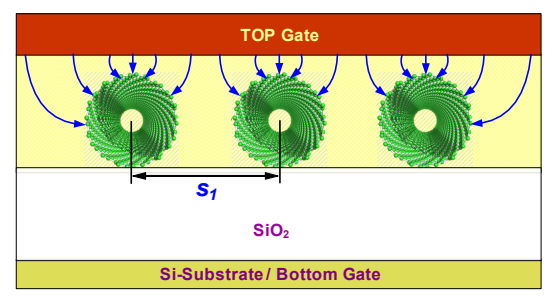

(c)

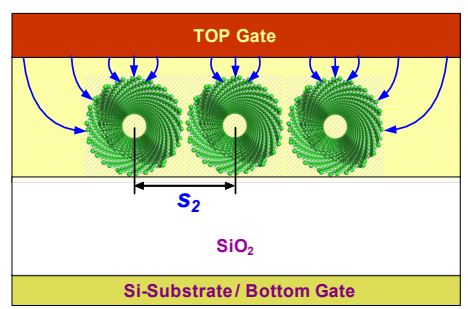

Fig. 4. The charge screening effect in an N-type multi-tube CN-MOSFET. A positive voltage is applied to the gate. (a) The channel contains only one nanotube. (b) The channel contains multiple tubes $(\mathrm{N}=3)$ with large inter-tube pitch $s_{1}$. (c) The channel contains multiple tubes $(\mathrm{N}=3)$ with a smaller inter-tube pitch $s_{2}$. As the pitch is reduced $\left(s_{2}\right.$ $<s_{1}$ ), electric field lines are screened and shielded by neighboring tubes, thereby lowering the screening effect coefficient $(\alpha)$ and the effective channel width $\left(W_{e f f}\right)$.

determined by the nanotube pitch. Screening effect is also influenced by the $\mathrm{CN}$ diameter, the number of tubes, the supply voltage, and the channel length of the device.

The current produced by a CN-MOSFET is determined by the effective channel width $W_{\text {eff. }} W_{\text {eff }}$ depends on the intensity of the electrical interactions among the carbon nanotubes that form the channel. Alternatively, the physical gate width $W_{g}$ depends only on the physical geometry of the device. $W_{g}$ and $W_{\text {eff }}$ are therefore different in a CN-MOSFET. Unlike a Si-MOSFET, $W_{g}$ contributes only to the parasitic capacitance and physical transistor area without directly influencing the drain current pro- 
duced by a CN-MOSFET.

\subsection{Device scaling and parameter settings}

The fixed (not considered for optimization) process parameters of the $16 \mathrm{~nm}$ CN-MOSFETs in this study are listed in Table 1. The 'Default value for $32 \mathrm{~nm}$ CN-MOSFET' column includes the default device parameters of the $32 \mathrm{~nm}$ Stanford University CNMOSFET technology presented in [12]. As listed in Table 1, the interconnect capacitance increases when the channel length is scaled [16]. High-performance nanotube transistors with integrated high-k ( 25) dielectrics (zirconium oxide thin-films) are described by Javey et al. in [18]. A high-k gate oxide material $\left(\mathrm{ZrO}_{2}\right)$ with a dielectric constant of 25 and a nominal thickness of $3 \mathrm{~nm}$ is assumed here to achieve high-speed transistors.

\section{OPTIMIZATION OF N-TYPE CN-MOSFET}

The current produced by a CN-MOSFET depends on important physical parameters such as the diameters of nanotubes, the inter-tube pitch, and the number of tubes. These parameters determine the effective channel width and device strength. In this section, the $\mathrm{CN}$ diameters are optimized to maximize the $I_{\text {on }} / I_{\text {off }}$ ratio for different nanotube arrays with different effective device channel widths. The influence of inter-tube pitch on device performance is evaluated with two different substrate (bottom-gate) voltages. The design tradeoffs among $\mathrm{I}_{\mathrm{on}}, \mathrm{I}_{\text {off }}$, and $\mathrm{I}_{\mathrm{on}} / \mathrm{I}_{\text {off }}$ for $\mathrm{N}$-type CN-MOSFETs are presented. All of the nanotubes are assumed to be semiconducting with uniform intertube pitch. Imperfections such as diameter variations and metallic nanotubes during the manufacturing of CN-MOSFETs are not considered in this paper. The die temperature is assumed to be $90^{\circ} \mathrm{C}[20]$. The nominal supply voltage is $0.7 \mathrm{~V}$. The channel lengths of all the CN-MOSFETs considered in this paper are 16 $\mathrm{nm}\left(\mathrm{L}_{\mathrm{g}}=16 \mathrm{~nm}\right)$.

\subsection{Nanotube diameter optimization}

In this section, the diameter of carbon nanotubes is optimized to achieve the maximum $\mathrm{I}_{\text {on }} / \mathrm{I}_{\text {off }}$ ratio. $\mathrm{I}_{\text {on }}$ is the drain current at $\mathrm{V}_{\mathrm{GS}}$ $=\mathrm{V}_{\mathrm{DS}}=\mathrm{V}_{\mathrm{DD}}=0.7 \mathrm{~V}$. $\mathrm{I}_{\text {off }}$ is the subthreshold leakage current at $\mathrm{V}_{\mathrm{GS}}=$ $0 \mathrm{~V}$ and $\mathrm{V}_{\mathrm{DS}}=\mathrm{V}_{\mathrm{DD}}=0.7 \mathrm{~V}$.

In order to evaluate the dependence of $\mathrm{I}_{\text {on }}$ and $\mathrm{I}_{\text {off }}$ on the diameter of nanotubes, an N-type CN-MOSFET with 2 tubes is presented as an example next. As discussed in Section 2.2, nanotube array pitch has a strong influence on $\mathrm{I}_{\mathrm{on}}$ produced by a CN-MOSFET. Similarly, the maximum achievable $\mathrm{I}_{\text {on }} / \mathrm{I}_{\text {off }}$ is affected by the array pitch. The optimum nanotube diameter that maximizes $I_{\text {on }} / I_{\text {off }}$ however is insensitive to the nanotube array pitch. The inter-tube pitch $(s)$ is assumed to be $1 \mu \mathrm{m}$ in this section to effectively eliminate the charge screening effect (i.e., $\alpha \approx 1$ ) and determine the maximum $\mathrm{I}_{\text {on }} / \mathrm{I}_{\text {off }}$ achievable with a CN-MOSFET. The maximum performance provided by an ideal CN transistor that does not suffer from charge screening effect is identified. The range of nanotube diameters is from $0.5 \mathrm{~nm}$ to $3 \mathrm{~nm}$. All the possible chirality vectors $(\mathrm{n}, \mathrm{m})$ that can produce the diameters in this range are considered in this study.

For a fixed number of tubes and a fixed pitch, enlarging the $\mathrm{CN}$ diameter enhances both $\mathrm{I}_{\text {on }}$ and $\mathrm{I}_{\text {off }}$ as shown in Fig. 5. The increased diameter decreases the resistance of the channel region. The source and drain resistances are also reduced due to the higher number of carriers induced in the semiconducting subbands as the diameter is enlarged. The variation of $\mathrm{I}_{\mathrm{on}}$ is primarily

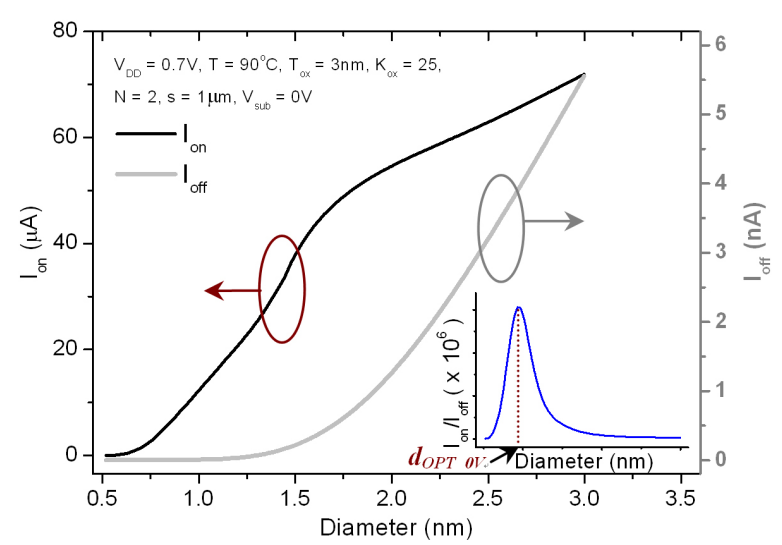

Fig. 5. The variation of $I_{o n}, I_{\text {off }}$, and $I_{\text {on }} / I_{\text {off }}$ with diameter for $N=2$. The substrate voltage $\left(\mathrm{V}_{\text {sub }}\right)=0 \mathrm{~V}$.

due to the strong modulation of the source and drain resistances with the CN diameter [21].

Subthreshold leakage current is exponentially increased with the diameter. $\mathrm{I}_{\text {off }}$ is controlled by the energy bandgap $\left(E_{g}\right)$ of CNs. $\mathrm{I}_{\text {off }}$ is dominated by the band-to-band tunneling current in a $\mathrm{CN}$ MOSFET [2]. The energy bandgap of $\mathrm{CN}$ is reduced as the diameter is increased. In the subthreshold region, particularly when a negative gate voltage is applied to an N-type CN-MOSFET, the band-to-band tunneling current is enhanced with increased diameter. The additional band-to-band leakage current $\left(\mathrm{I}_{\mathrm{btbt}}\right)$ through the semiconducting sub-bands is particularly severe for high $\mathrm{V}_{\mathrm{DS}}$ in a CN-MOSFET [21].

When the diameter $\left(d_{C N}\right)$ is increased, $\mathrm{I}_{\text {on }}$ and $\mathrm{I}_{\text {off }}$ are enhanced at different rates. The $\mathrm{I}_{\mathrm{on}} / \mathrm{I}_{\mathrm{off}}$ is therefore maximized at an optimum diameter $\left(d_{O P T_{-} O V}\right)$ as shown in Figs. 5 and 6 with $0 \mathrm{~V}$ substrate bias. Similar trends for the variation of $\mathrm{I}_{\mathrm{on}}, \mathrm{I}_{\mathrm{off}}$, and $\mathrm{I}_{\mathrm{on}} / \mathrm{I}_{\text {off }}$ with the diameter are observed when the substrate is connected to the power supply $(0.7 \mathrm{~V})$ as shown in Fig. 6 . The $\mathrm{x}$-axis of Fig. 6 corresponds to $\mathrm{I}_{\mathrm{on}}$ when the diameter is increased from $0.5 \mathrm{~nm}$ to $3 \mathrm{~nm}$.

The substrate can be viewed as a second (bottom) gate below a thick oxide layer in a CN-MOSFET [12]. $\mathrm{I}_{\text {on }}$ is enhanced due to stronger channel inversion with a higher substrate voltage. The subthreshold leakage current however is also increased when the substrate voltage is higher than $0 \mathrm{~V}$. An n-channel CN-MOSFET cannot be effectively cut-off provided that the substrate is connected to the power supply $\left(\mathrm{V}_{\mathrm{sub}}=\mathrm{V}_{\mathrm{DD}}=0.7 \mathrm{~V}\right)$. The maximum achievable $\mathrm{I}_{\mathrm{on}} / \mathrm{I}_{\text {off }}$ and the corresponding optimum nanotube diameter are therefore reduced with a higher substrate voltage.

The variation of maximum achievable $\mathrm{I}_{\text {on }} / \mathrm{I}_{\text {off }}$ with the number of tubes $(1 \leq N \leq 35)$ for two different substrate voltages is shown in Fig. 7. For each number of tubes, the percent enhancement of the maximum achievable $\mathrm{I}_{\text {on }} / \mathrm{I}_{\text {off }}$ by decreasing the substrate voltage from $0.7 \mathrm{~V}$ to $0 \mathrm{~V}$ is also shown. By connecting the substrate to the ground, the maximum $\mathrm{I}_{\text {on }} / \mathrm{I}_{\text {off }}$ is enhanced by up to $43 \%$ as compared to the maximum $\mathrm{I}_{\text {on }} / \mathrm{I}_{\text {off }}$ achievable with a higher substrate voltage.

\subsection{The effect of substrate voltage on the optimum diameter}

The variation of optimum nanotube diameter with the transistor size (number of tubes) for $\mathrm{V}_{\text {sub }}=0 \mathrm{~V}$ and $\mathrm{V}_{\text {sub }}=0.7 \mathrm{~V}$ is shown in Fig. 8. Both $d_{O P T_{-} O V}$ and $d_{O P T_{-} 0.7 V}$ are reduced with the increased $\mathrm{N}$ as shown in Fig. 8. When $N$ is increased from 1 to $35, d_{O P T_{-} 0.7 V}$ is reduced from $0.872 \mathrm{~nm}$ to $0.691 \mathrm{~nm}$ (a reduction of approximately $26 \%$ ). Alternatively, when $\mathrm{V}_{\text {sub }}=0 \mathrm{~V}, d_{O P T_{-} O V}$ is reduced from 


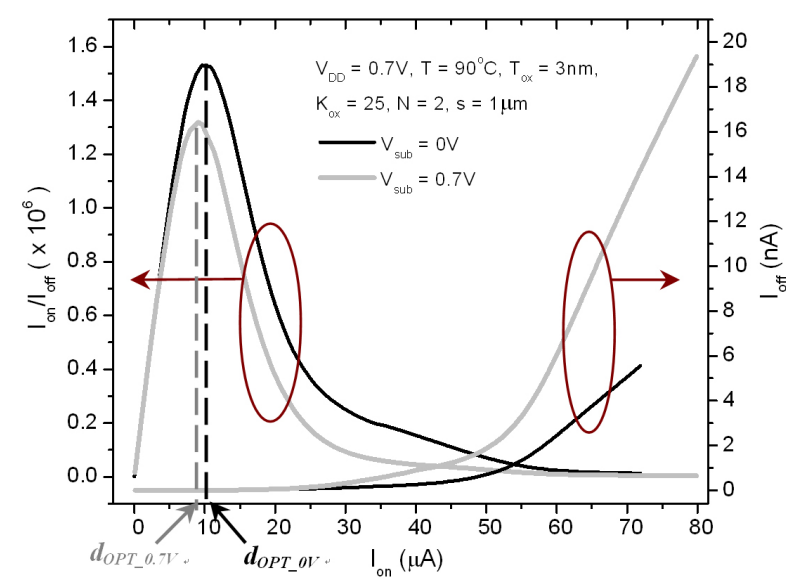

Fig. 6. The variations of $\mathrm{I}_{\text {off }}$ and $\mathrm{I}_{\text {on }} / \mathrm{I}_{\text {off }}$ with $\mathrm{I}_{\text {on }}$ for two different substrate voltages $(0 \mathrm{~V}$ and $0.7 \mathrm{~V}) . \mathrm{N}=2$.

$0.993 \mathrm{~nm}$ to $0.804 \mathrm{~nm}$ (a reduction of approximately 23\%) with the increased transistor size (as $N$ is increased from 1 to 35 ).

In addition to enhanced $\mathrm{I}_{\text {on }} / \mathrm{I}_{\text {off }}$, the optimum nanotube diameter that maximizes $\mathrm{I}_{\mathrm{on}} / \mathrm{I}_{\mathrm{off}}$ is also enlarged with a smaller substrate voltage. For $1 \leq N \leq 35, d_{O P T_{-} O V}$ is $7.4 \%$ to $16.4 \%$ larger as compared to $d_{O P T_{-} 0.7 V}$ as shown in Fig. 8. A lower substrate voltage with a larger optimum diameter may be preferable for easier manufacturability. Furthermore, the increased $d_{\text {OPT ov }}$ has little influence on the device physical gate width $\left(W_{g}\right)$ and area. $W_{g}$ is typically dominated by the gate overhang width, the nanoarray pitch, and the number of nanotubes in a CN-MOSFET.

\subsection{Device performance versus integration density tradeoffs}

The ideal maximum $\mathrm{I}_{\mathrm{on}} / \mathrm{I}_{\text {off }}$ of an optimized N-type CN-MOSFET is achieved at a high inter-tube pitch (assumed to be $1 \mu \mathrm{m}$ in Section 3.1) where the screening effect is negligible. This pitch is however impractically long from an area efficiency point of view. Shorter pitches are desirable to enhance the integration density of a chip with CN-MOSFET technology. In this section, the effect of pitch reduction on the maximum achievable $\mathrm{I}_{\mathrm{on}} / \mathrm{I}_{\text {off }}$ is evaluated.

As shown in Fig. 9 with $N=2$, the optimum diameter $d_{O P T}$ is independent of the inter-tube pitch $(s)$. The optimum diameters are maintained similar to the values illustrated in Fig. 8 when the pitch is scaled for various transistor sizes $(1 \leq N \leq 35)$. As listed in Tables 2 and 3, for each $N$, four (relatively more practical from an area efficiency point of view) pitch values $(s)$ and the corresponding physical gate widths $\left(W_{g}\right)$ are identified with two different substrate voltages. $5 \%, 10 \%, 15 \%$, and $20 \%$ degradations from the ideal maximum $\mathrm{I}_{\mathrm{on}} / \mathrm{I}_{\text {off }}$ (that could only be achieved by an essentially ideal switch with an impractically large pitch and negligible charge screening effect) are assumed to be acceptable for implementing high performance and compact integrated circuits.

As $N$ is increased from 1 to 35 , both optimum $\mathrm{I}_{\text {on }} / \mathrm{I}_{\text {off }}$ and $W_{g}$ are increased. For a fixed $N$, shorter $s$ is desirable for a smaller device area $\left(W_{g}\right.$ is reduced). However, the effective channel width $\left(W_{e f f}\right)$ is also reduced due to the enhanced charge screening effect (lower $\alpha$ ) with a shorter nanotube pitch (see Eq. (4)). The $\mathrm{I}_{\text {on }}$ therefore decreases with a shorter $s$. The variation of the subthreshold leakage current with the pitch is negligible since $\mathrm{I}_{\text {off }}$ is primarily controlled by the $\mathrm{E}_{\mathrm{g}}$ and $\mathrm{I}_{\mathrm{btbt}}$. Lowering the pitch to reduce the device area degrades $\mathrm{I}_{\text {on }} / \mathrm{I}_{\text {off }}$ as listed in Tables

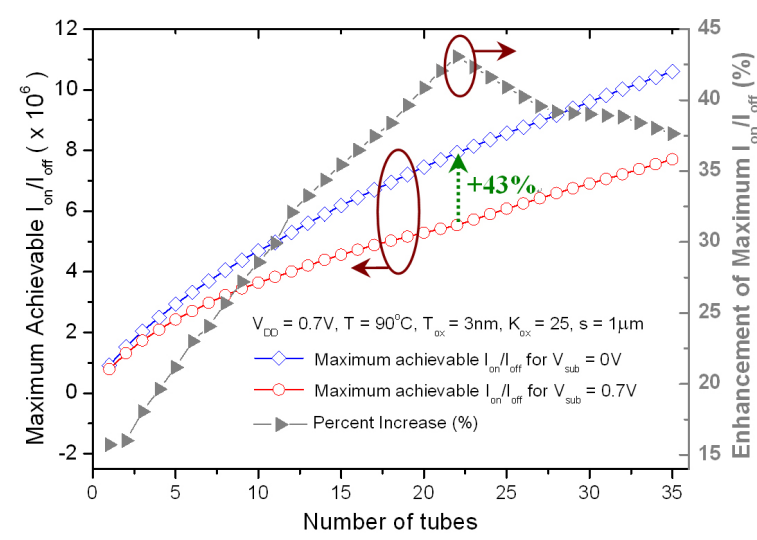

Fig. 7. The variations of maximum achievable $\mathrm{I}_{\mathrm{on}} / \mathrm{I}_{\text {off }}$ with the number of tubes for two different substrate voltages $(0 \mathrm{~V}$ and $0.7 \mathrm{~V})$. The percent enhancement of maximum achievable $\mathrm{I}_{\text {on }} / \mathrm{I}_{\text {off }}$ by lowering the substrate bias voltage from $0.7 \mathrm{~V}$ to $0 \mathrm{~V}$ is also shown.

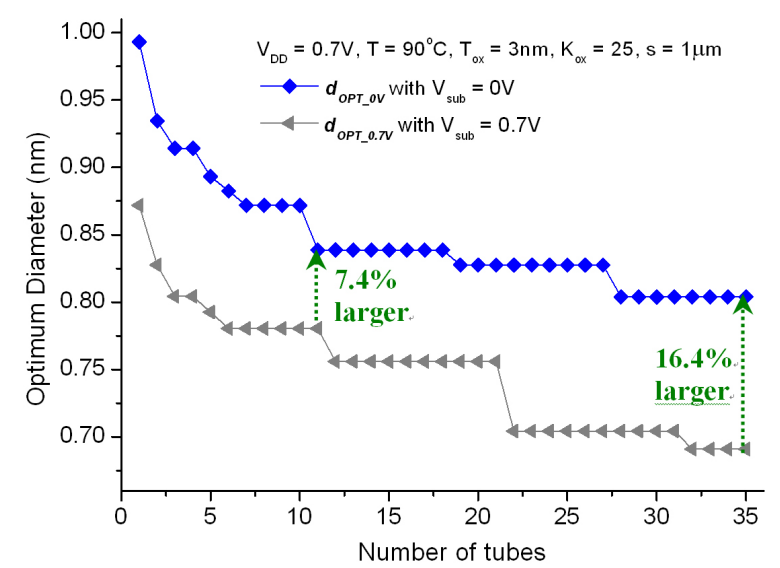

Fig. 8. The variation of optimum diameter with transistor size (number of tubes) for two different substrate voltages $(0 \mathrm{~V}$ and $0.7 \mathrm{~V})$.

2 and 3. There is therefore a tradeoff between switch performance $\left(\mathrm{I}_{\mathrm{on}} / \mathrm{I}_{\text {off }}\right)$ and area efficiency $\left(W_{\mathrm{g}}\right)$. Alternatively, as listed in Tables 2 and 3 and as discussed in Section 3.1, for the same number of tubes and similar physical gate width, the maximum achievable $\mathrm{I}_{\text {on }} / \mathrm{I}_{\text {off }}$ is enhanced with $\mathrm{V}_{\text {sub }}=0 \mathrm{~V}$ as compared to $\mathrm{V}_{\text {sub }}=0.7 \mathrm{~V}$. A smaller substrate voltage is therefore desirable to enhance $I_{o n} / I_{\text {off }}$ without degrading the integration density of $n$ channel CN-MOSFETs.

\subsection{Uniform nanotube diameter for manufacturabil- ity with different transistor sizes}

As shown in Sections 3.1 and 3.2, $\mathrm{I}_{\text {on }} / \mathrm{I}_{\text {off }}$ can be maximized by manufacturing nanotubes with carefully optimized diameters $d_{O P T}$ for achieving high-speed and low-power integrated circuits. Billions of transistors with various driving strengths (various device widths) will be required to implement complex state-of-theart integrated circuits with the carbon nanotube technology. The strength of CN-MOSFETs can be tuned by adjusting the number of tubes that form the channel as explained in Section 2. The optimum diameters are listed in Tables 2 and 3 for various sizes of N-type CN-MOSFETs with different numbers of tubes $(1 \leq N \leq$ 35).

The optimum diameter for achieving the maximum $\mathrm{I}_{\text {on }} / \mathrm{I}_{\text {off }}$ varies with the number of tubes (transistor size) as shown in Fig. 8. 
Table 2. Pitch values for $5 \%, 10 \%, 15 \%$, and $20 \%$ degradation from the maximum achievable $\mathrm{I}_{\mathrm{ON}} / \mathrm{I}_{\mathrm{OFF}}$ with $16 \mathrm{~nm} \mathrm{~N}$-type CN-MOSFETs. $\mathrm{T}=90^{\circ} \mathrm{C}$. Substrate voltage $=0 \mathrm{~V}$.

\begin{tabular}{|c|c|c|c|c|c|c|c|c|c|c|c|}
\hline \multirow{2}{*}{$\begin{array}{l}\text { Number } \\
\text { of tubes } \\
(N)^{\mathrm{a}}\end{array}$} & \multirow{2}{*}{$\begin{array}{l}\text { Optimum } \\
\text { diameter } \\
d_{O P T_{-} O V} \\
(\mathrm{~nm})\end{array}$} & \multirow{2}{*}{$\begin{array}{l}\text { Optimum } \\
\text { chirality vec- } \\
\text { tor }(n, m)\end{array}$} & \multirow{2}{*}{$\begin{array}{l}\text { Maximum } \\
\mathrm{I}_{\text {on }} / \mathrm{I}_{\text {off }} \\
\left(\times 10^{6}\right) \\
\text { at } s=1 \mu \mathrm{m}\end{array}$} & \multicolumn{2}{|c|}{$\begin{array}{l}\text { With } 5 \% \text { degradation } \\
\text { from maximum } I_{o n} / I_{o f f}\end{array}$} & \multicolumn{2}{|c|}{$\begin{array}{l}\text { With } 10 \% \text { degradation } \\
\text { from maximumI } \mathrm{on}_{\text {on }} / \mathrm{I}_{\text {off }}\end{array}$} & \multicolumn{2}{|c|}{$\begin{array}{l}\text { With } 15 \% \text { degradation } \\
\text { from maximum } I_{\text {on }} / I_{\text {off }}\end{array}$} & \multicolumn{2}{|c|}{$\begin{array}{l}\text { With } 20 \% \text { degradation } \\
\text { from maximum } I_{\text {on }} / I_{\text {off }}\end{array}$} \\
\hline & & & & $s(\mathrm{~nm})$ & $W_{g}(\mathrm{~nm})$ & $s(\mathrm{~nm})$ & $W_{g}(\mathrm{~nm})$ & $s(\mathrm{~nm})$ & $W_{g}(\mathrm{~nm})$ & $s(\mathrm{~nm})$ & $W_{g}(\mathrm{~nm})$ \\
\hline 1 & 0.993 & $(1,12),(12,1)$ & 0.913 & -- & 32.99 & -- & 32.99 & -- & 32.99 & -- & 32.99 \\
\hline 2 & 0.934 & $(3,10),(10,3)$ & 1.530 & 10.64 & 43.57 & 7.05 & 39.98 & 5.35 & 38.28 & 4.27 & 37.20 \\
\hline 6 & 0.883 & $(2,10),(10,2)$ & 3.327 & 14.09 & 103.33 & 9.64 & 81.08 & 7.6 & 70.88 & 6.32 & 64.48 \\
\hline 10 & 0.872 & $(0,11),(11,0)$ & 4.688 & 14.65 & 164.72 & 10.05 & 123.32 & 7.94 & 104.33 & 6.64 & 92.63 \\
\hline 14 & 0.839 & $(4,8),(8,4)$ & 5.896 & 14.81 & 225.37 & 10.17 & 165.05 & 8.04 & 137.36 & 6.72 & 120.20 \\
\hline 18 & 0.839 & $(4,8),(8,4)$ & 6.949 & 14.94 & 286.82 & 10.27 & 207.43 & 8.12 & 170.88 & 6.8 & 148.44 \\
\hline 22 & 0.828 & $(5,7),(7,5)$ & 7.919 & 14.98 & 347.41 & 10.29 & 248.92 & 8.14 & 203.77 & 6.82 & 176.05 \\
\hline 26 & 0.828 & $(5,7),(7,5)$ & 8.766 & 15.04 & 408.83 & 10.34 & 291.33 & 8.18 & 237.33 & 6.85 & 204.08 \\
\hline 30 & 0.804 & $(2,9),(9,2)$ & 9.598 & 14.96 & 466.64 & 10.28 & 330.92 & 8.13 & 268.57 & 6.82 & 230.58 \\
\hline 35 & 0.804 & $(2,9),(9,2)$ & 10.600 & 15.01 & 543.14 & 10.31 & 383.34 & 8.16 & 310.24 & 6.84 & 265.36 \\
\hline
\end{tabular}

${ }^{a}$ All the device sizes for $1 \leq \mathrm{N} \leq 35$ are considered in this optimization study. Only a small number of selected devices are listed in the table due to limited space.

Table 3. Pitch values for $5 \%, 10 \%, 15 \%$, and $20 \%$ degradation from the maximum achievable $\mathrm{I}_{\mathrm{ON}} / \mathrm{I}_{\mathrm{OFF}}$ with $16 \mathrm{~nm} \mathrm{~N}-\mathrm{type} \mathrm{CN}-\mathrm{MOSFETs}$. $\mathrm{T}=90^{\circ} \mathrm{C}$. Substrate voltage $=0.7 \mathrm{~V}$.

\begin{tabular}{|c|c|c|c|c|c|c|c|c|c|c|c|}
\hline \multirow{2}{*}{$\begin{array}{l}\text { Number } \\
\text { of tubes } \\
(N)^{\mathrm{a}}\end{array}$} & \multirow{2}{*}{$\begin{array}{l}\text { Optimum } \\
\text { diameter } \\
d_{\text {OPT_0.7V }} \\
(\mathrm{nm})\end{array}$} & \multirow{2}{*}{$\begin{array}{l}\text { Optimum } \\
\text { chirality vec- } \\
\text { tor }(n, m)\end{array}$} & \multirow{2}{*}{$\begin{array}{l}\text { Maximum } \\
\mathrm{I}_{\mathrm{on}} / \mathrm{I}_{\text {off }} \\
\left(\times 10^{6}\right) \\
\text { at } s=1 \mu \mathrm{m}\end{array}$} & \multicolumn{2}{|c|}{$\begin{array}{l}\text { With } 5 \% \text { degradation } \\
\text { from maximum } I_{\text {on }} / I_{\text {off }}\end{array}$} & \multicolumn{2}{|c|}{$\begin{array}{l}\text { With } 10 \% \text { degradation } \\
\text { from maximumI } \mathrm{o}_{\text {on }} / \mathrm{I}_{\text {off }}\end{array}$} & \multicolumn{2}{|c|}{$\begin{array}{l}\text { With } 15 \% \text { degradation } \\
\text { from maximum } \mathrm{I}_{\text {on }} / \mathrm{I}_{\text {off }}\end{array}$} & \multicolumn{2}{|c|}{$\begin{array}{l}\text { With } 20 \% \text { degradation } \\
\text { from maximum } \mathrm{I}_{\text {on }} / \mathrm{I}_{\text {off }}\end{array}$} \\
\hline & & & & $s(\mathrm{~nm})$ & $W_{g}(\mathrm{~nm})$ & $s(\mathrm{~nm})$ & $W_{g}(\mathrm{~nm})$ & $s(\mathrm{~nm})$ & $W_{g}(\mathrm{~nm})$ & $s(\mathrm{~nm})$ & $W_{g}(\mathrm{~nm})$ \\
\hline 1 & 0.872 & $(0,11),(11,0)$ & 0.789 & -- & 32.87 & -- & 32.87 & -- & 32.87 & -- & 32.87 \\
\hline 2 & 0.828 & $(5,7),(7,5)$ & 1.319 & 11.06 & 43.89 & 7.4 & 40.23 & 5.7 & 38.53 & 5.42 & 38.25 \\
\hline 6 & 0.781 & $(3,8),(8,3)$ & 2.705 & 14.7 & 106.28 & 10.17 & 83.63 & 8.12 & 73.38 & 6.86 & 67.08 \\
\hline 10 & 0.781 & $(3,8),(8,3)$ & 3.646 & 16.77 & 183.71 & 11.64 & 137.54 & 9.31 & 116.57 & 7.89 & 103.79 \\
\hline 14 & 0.756 & $\begin{array}{l}(1,9),(9,1) \\
(5,6),(6,5)\end{array}$ & 4.388 & 16.06 & 241.54 & 11.15 & 177.71 & 8.92 & 148.72 & 7.56 & 131.04 \\
\hline 18 & 0.756 & $\begin{array}{l}(1,9),(9,1) \\
(5,6),(6,5)\end{array}$ & 5.022 & 16.95 & 320.91 & 11.78 & 233.02 & 9.42 & 192.90 & 7.99 & 168.59 \\
\hline 22 & 0.705 & $(3,7),(7,3)$ & 5.536 & 13.14 & 308.65 & 9.10 & 223.81 & 7.28 & 185.59 & 6.17 & 162.28 \\
\hline 26 & 0.705 & $(3,7),(7,3)$ & 6.252 & 13.69 & 374.96 & 9.49 & 269.96 & 7.59 & 222.46 & 6.44 & 193.71 \\
\hline 30 & 0.705 & $(3,7),(7,3)$ & 6.906 & 14.17 & 443.64 & 9.82 & 317.49 & 7.86 & 260.65 & 6.67 & 226.14 \\
\hline 35 & 0.691 & $(4,6),(6,4)$ & 7.702 & 13.47 & 490.67 & 9.34 & 350.25 & 7.46 & 286.33 & 6.33 & 247.91 \\
\hline
\end{tabular}

${ }^{a}$ All the device sizes for $1 \leq \mathrm{N} \leq 35$ are considered in this optimization study. Only a small number of selected devices are listed in the table due to limited space.

Fabricating nanotubes with different diameters for various sizes of CN-MOSFETs on a complex chip is not practical. For low-cost and high-yield manufacturability, it is highly desirable to have only one uniform nanotube diameter (for a single- $\mathrm{V}_{\text {th }} \mathrm{CN}-\mathrm{MOS}$ FET technology) across a chip. The effect of a uniform diameter on the performance of various sizes of CN-MOSFETs is evaluated in this section. 35 different transistor sizes are considered (the number of tubes is varied from 1 to 35) to determine a single uniform and manufacturable diameter with acceptable degradation from the ideal switch performance.

Three possible uniform diameters are evaluated for various sizes of CN-MOSFETs. The average diameter $\left(d_{A V G}\right)$ is the average value of the optimum nanotube diameters shown in Fig. 8 for $1 \leq N \leq 35$. The maximum diameter $\left(d_{M A X}\right)$ is the maximum $d_{O P T}$ determined for $1 \leq N \leq 35$. Alternatively, the minimum diameter $\left(d_{M I N}\right)$ is the minimum $d_{O P T}$ in Fig. 8 for $1 \leq N \leq 35$. The $d_{M A X}$ and $d_{M I N}$ are observed for $N=1$ and $N=35$, respectively, since $d_{O P T}$ that maximizes $\mathrm{I}_{\text {on }} / \mathrm{I}_{\text {off }}$ is reduced with increased $N$ as illustrated in Fig. 8.

The degradation from the ideal maximum $\mathrm{I}_{\text {on }} / \mathrm{I}_{\text {off }}$ (observed at $\left.d_{O P T_{-} O V}\right)$ for $d_{A V G_{-} O V}, d_{\text {MAX_OVV }}$, and $d_{\text {MIN_OV }}$ with different transistor sizes is shown in Fig. 10 when $V_{\text {sub }}=0 \mathrm{~V}$. By using nanotubes with a uniform diameter of $d_{A V G_{-} O V}(0.839 \mathrm{~nm})$, the degradation from the ideal maximum $\mathrm{I}_{\text {on }} / \mathrm{I}_{\text {off }}$ is maintained below $20 \%$ for $3 \leq$ $N \leq 35$. Further analysis for even larger transistors reveals that $\mathrm{I}_{\text {on }} / \mathrm{I}_{\text {off }}$ degradations are maintained below the $20 \%$ demarcation line by employing a uniform diameter of $0.839 \mathrm{~nm}$ for $36 \leq$ $N \leq 73$ as well. For a single- $\mathrm{V}_{\text {th }} \mathrm{CN}$ transistor technology where only one uniform nanotube diameter is desirable for low-cost and high-yield manufacturability, the suggested nanotube diameter is therefore $0.839 \mathrm{~nm}$ for $\mathrm{n}$-channel CN-MOSFETs with $\mathrm{V}_{\text {sub }}=0 \mathrm{~V}$. For $N<3$, a diameter larger than $d_{A V G_{-} O V}$ is desirable for higher $\mathrm{I}_{\text {on }} / \mathrm{I}_{\text {off }}$. Alternatively, for $N>73$, a diameter smaller than $d_{A V G_{0} O V}$ is desirable to limit $\mathrm{I}_{\text {on }} / \mathrm{I}_{\text {off }}$ degradations to less than $20 \%$.

Similar analysis is also conducted to determine a practical uniform diameter for which the $\mathrm{I}_{\mathrm{on}} / \mathrm{I}_{\text {off }}$ degradations are smaller than $20 \%$ at $\mathrm{V}_{\text {sub }}=0.7 \mathrm{~V}$. As shown in Fig. 11, the performance degradations are maintained below $20 \%$ by using nanotubes with a uniform diameter of $d_{A V G_{\_} . .7 V}(0.756 \mathrm{~nm})$ for $3 \leq N \leq 43$. Therefore, the suggested uniform nanotube diameter is $0.756 \mathrm{~nm}$ for $\mathrm{V}_{\text {sub }}=0.7$ V. For $N<3$, a diameter larger than $d_{A V G \text { 0.7V }}$ is desirable for higher $\mathrm{I}_{\text {on }} / \mathrm{I}_{\text {off }}$. Alternatively, for $N>43$, a diameter smaller than $d_{A V G_{-} 0.7 V}$ is desirable to maintain $\mathrm{I}_{\text {on }} / \mathrm{I}_{\text {off }}$ degradations below $20 \%$. 


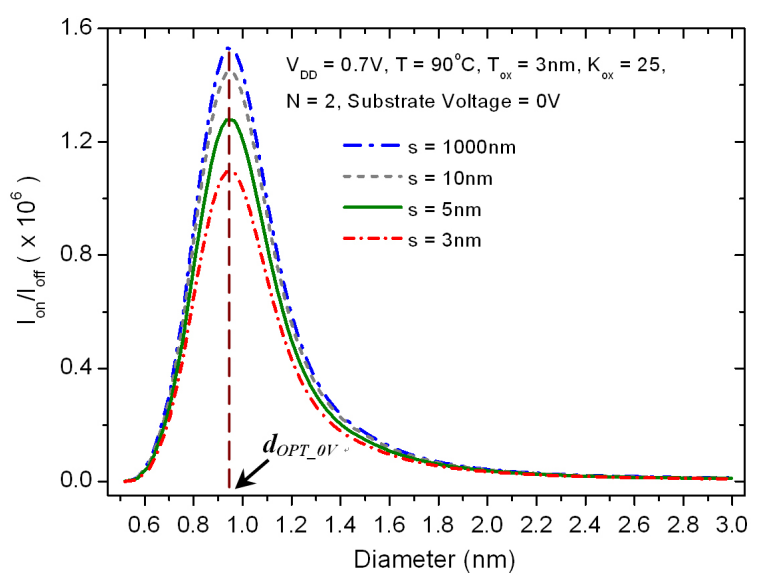

Fig. 9. The variation of $\mathrm{I}_{\mathrm{on}} / \mathrm{I}_{\text {off }}$ with the diameter for different pitch values $(s)$. The optimum diameter that maximizes $\mathrm{I}_{\text {on }} / \mathrm{I}_{\text {off }}$ is insensitive to pitch variations. The substrate voltage $=0 \mathrm{~V}$.

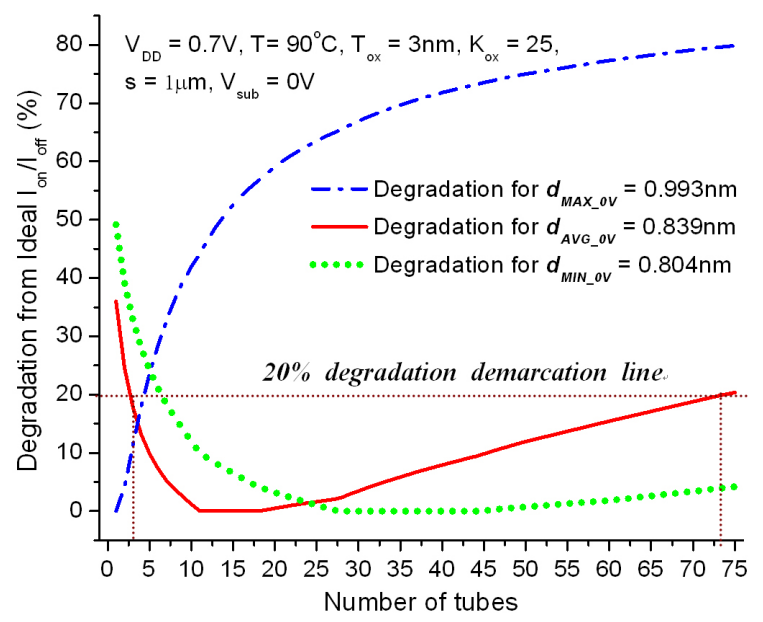

Fig. 10. The degradation from the ideal maximum $\mathrm{I}_{\text {on }} / \mathrm{I}_{\text {off }}$ (observed at $d_{\text {OPT_OV }}$ ) for $d_{\text {AVG_OV }}, d_{\text {MAX_OV }}$, and $d_{\text {MIN_OV }}$ with different number of tubes when the substrate voltage $=0 \mathrm{~V}$. The $20 \%$ degradation from the ideal maximum $I_{o n} / I_{\text {off }}$ is demarcated with a dashed line. The performance degradations of devices below the demarcation line are less than $20 \%$ as compared to the ideal maximum $\mathrm{I}_{\text {on }} / \mathrm{I}_{\text {off }}$ values at $\mathrm{V}_{\text {sub }}=0 \mathrm{~V}$.

\section{CONCLUSIONS}

The influence of device physical parameters on the electrical characteristics of $16 \mathrm{~nm} \mathrm{~N}$-type CN-MOSFETs is explored in this paper. The optimum $16 \mathrm{~nm}$ device profiles are identified for different nanotube arrays at $90^{\circ} \mathrm{C}$. Design guidelines are provided for the development and accurate characterization of highspeed, low-power, and compact integrated circuits with carbonnanotube transistors.

The nanotube diameter, the inter-tube pitch, and the number of tubes per device play the most important roles in determining both the area (the physical gate width) and the performance $\left(\mathrm{I}_{\text {on }} / \mathrm{I}_{\text {off }}\right)$ of carbon-nanotube transistors. Tradeoffs among area efficiency, $\mathrm{I}_{\mathrm{on}}$, and $\mathrm{I}_{\mathrm{on}} / \mathrm{I}_{\text {off }}$ of $\mathrm{N}$-type CN-MOSFETs are explored in this paper. Furthermore, the influence of substrate voltage on device performance is investigated. For a higher number of tubes (larger transistor size), the optimum diameter that maximizes $\mathrm{I}_{\mathrm{on}} / \mathrm{I}_{\text {off }}$ is reduced. For a manufacturable, high-yield, and low-cost integrated circuit however only one uniform nanotube diameter

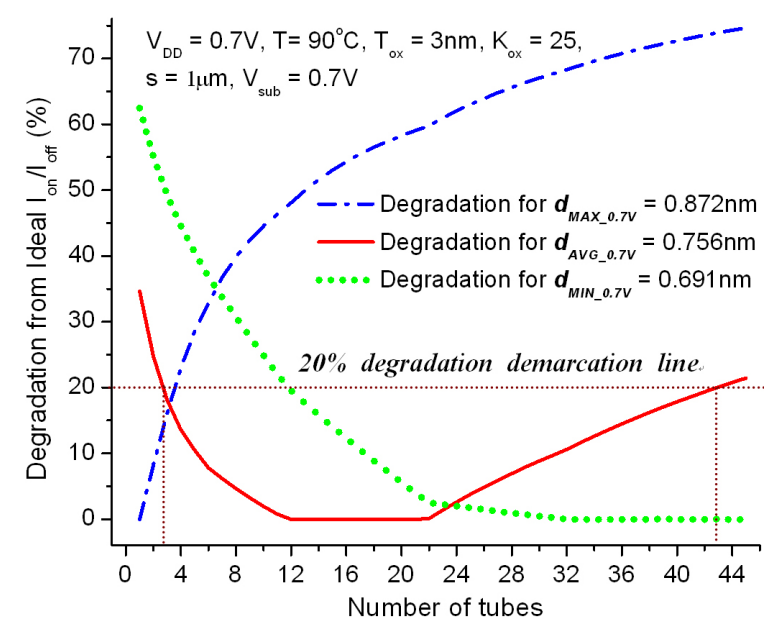

Fig. 11. The degradation from the ideal maximum $\mathrm{I}_{\text {on }} / \mathrm{I}_{\text {off }}$ (observed at $\left.d_{\text {OPT_ } 0.7 V}\right)$ for $d_{A V V_{-} 0.7 V}, d_{\text {MAX } 0.7 V}$, and $d_{\text {MIN_ } 0.7 V}$ with different number of tubes when the substrate voltage $=0.7 \mathrm{~V}$. The $20 \%$ degradation from the ideal maximum $\mathrm{I}_{\text {on }} / \mathrm{I}_{\text {off }}$ is demarcated with a dashed line. The performance degradations of devices below the demarcation line are less than $20 \%$ as compared to the ideal maximum $\mathrm{I}_{\text {on }} / \mathrm{I}_{\text {off }}$ values at $\mathrm{V}_{\text {sub }}$ $=0.7 \mathrm{~V}$.

is desirable across a chip. The degradation from the maximum achievable $\mathrm{I}_{\text {on }} / \mathrm{I}_{\text {off }}$ is maintained below $20 \%$ with a uniform diameter of $0.839 \mathrm{~nm}$ for $3 \leq N \leq 73$ when the substrate is connected to the ground. Alternatively, if the substrate is connected to the power supply, the suggested uniform nanotube diameter for high-performance and manufacturability is $0.756 \mathrm{~nm}$ for $3 \leq N \leq$ 43.

Either increasing the diameter beyond the optimum or increasing the substrate voltage of an N-type CN-MOSFET enhances the $\mathrm{I}_{\mathrm{on}}$. This speed enhancement is however achieved at the cost of higher leakage current and degraded $\mathrm{I}_{\text {on }} / \mathrm{I}_{\text {off. }}$. The $\mathrm{I}_{\text {on }} / \mathrm{I}_{\text {off }}$ can be enhanced by increasing the pitch (weakening the charge screening effect). A larger pitch however degrades the integration density. The tradeoffs between $\mathrm{I}_{\text {on }} / \mathrm{I}_{\text {off }}$ and area efficiency are highlighted with this study. Practical pitch values for achieving high performance within $5 \%, 10 \%, 15 \%$, and $20 \%$ of an ideal switch are identified.

\section{REFERENCES}

[1] P. Avouris, Z. Chen, and V. Perebeinos, Nature Nanotechnol. 2, 605 (2007) [DOI: 10.1038/nnano.2007.300].

[2] J. Guo, A. Javey, H. Dai, S. Datta, and M. Lundstrom, Predicted performance advantages of carbon nanotube transistors with doped nanotubes as source/drain (2003). Available from: http://arxiv.org/abs/cond-mat/0309039.

[3] J. Guo, A. Javey, H. Dai, and M. Lundstrom, IEEE International Electron Devices Meeting (San Francisco, CA 2004) p. 703.

[4] A. Javey, J. Guo, D. B. Farmer, Q. Wang, E. Yenilmez, R. G. Gordon, M. Lundstrom, and H. Dai, Nano Lett. 4, 1319 (2004) [DOI: $10.1021 /$ nl049222b].

[5] A. Javey, J. Guo, D. B. Farmer, Q. Wang, D. Wang, R. G. Gordon, M. Lundstrom, and H. Dai, Nano Lett. 4, 447 (2004) [DOI: 10.1021/ nl035185x].

[6] A. Javey, R. Tu, D. B. Farmer, J. Guo, R. G. Gordon, and H. Dai, Nano Lett. 5, 345 (2005) [DOI: 10.1021/nl047931j].

[7] S. Lin, Y. B. Kim, and F. Lombardi, IEEE Trans. Nanotechnol. 9, 30 (2010) [DOI: 10.1109/tnano.2009.2025128].

[8] M. Moradinasab, F. Karbassian, and M. Fathipour, 1st Asia Sym- 
posium on Quality Electronic Design (Kuala Lumpur 2009) p. 19

[9] B. Ebrahimi and A. Afzali-Kusha, 1st Asia Symposium on Quality Electronic Design (Kuala Lumpur 2009) p. 14.

[10] Y. B. Kim, F. Lombardi, and Y. J. Lee, International SoC Design Conference (Busan, Korea 2008) p. I176.

[11] S. Lin, Y. B. Kim, and F. Lombardi, 52nd IEEE International Midwest Symposium on Circuits and Systems (Cancun 2009) p. 435.

[12] Stanford University Nanoeletronics Group. Stanford University CNFET Model. Available: http://nano.stanford.edu/model. php?id=23.

[13] Y. Sun and V. Kursun, International SoC Design Conference (Incheon, Korea 2010) p. 260.

[14] R. Saito, G. Dresselhaus, and M. S. Dresselhaus, Physical Prop erties of Carbon Nanotubes (Imperial College Press, London, 1998).

[15] The MOSIS Service. MOSIS Scalable CMOS (SCMOS). Available: http://www.mosis.com/Technical/Designrules/scmos/scmos- main.html

[16] J. Deng and H. S. P. Wong, IEEE Trans. Electron Devices 54, 3186 (2007) [DOI: 10.1109/ted.2007.909030].

[17] C. Kshirsagar, H. Li, T. E. Kopley, and K. Banerjee, IEEE Electron Device Lett. 29, 1408 (2008) [DOI: 10.1109/led.2008.2007598].

[18] A. Javey, H. Kim, M. Brink, Q. Wang, A. Ural, J. Guo, P. McIntyre, P. McEuen, M. Lundstrom, and H. Dai, Nature Mater. 1, 241 (2002) [DOI: 10.1038/nmat769].

[19] Y. Lu, S. Bangsaruntip, X. Wang, L. Zhang, Y. Nishi, and H. Dai, J. Am. Chem. Soc. 128, 3518 (2006) [DOI: 10.1021/ja058836v].

[20] B. Black, M. Annavaram, N. Brekelbaum, J. Devale, J. Lei, G. H. Loh, D. McCauley, P. Morrow, D. W. Nelson, D. Pantuso, P. Reed, J. Rupley, S. Shankar, S. John, and C. Webb, 39th Annual IEEE/ ACM International Symposium on Microarchitecture (Orlando, FL 2006) p. 469-479.

[21] J. Deng and H. S. P. Wong, IEEE Trans. Electron Devices 54, 3195 (2007) [DOI: 10.1109/ted.2007.909043]. 